\title{
Entre la decolonización y el capital humano. reflexiones en torno al rol esperado del docente en el Chile post transición
}

\section{Between Decolonization and Human Capital. Reflections Toward the Expected Role of the Teacher in the Chile Post Transition}

Patricia E. Troncoso-Pérez

Universidad del Bío-Bío, Chillán, Chile

Universidad Adventista de Chile, Chillan, Chile

ORCID: https://orcid.org/0000-0002-6926-6427

Juan Luis Nass Álvarez

Universidad Católica de Temuco, Chile

ORCID: https://orcid.org/0000-0001-8644-1672

\section{Ada Gallegos}

Universidad Nacional Mayor de San Marcos, Perú ORCID: https://orcid.org/0000-0002-8264-711X

\section{Miguel A. Alvarez}

Universidad de las Américas, Chile

ORCID: https://orcid.org/0000-0002-3893-2204

Recibido 02-01-20 Revisado 15-02-20 Aprobado 30-03-20 En línea 07-04-20

*Correspondencia

Email: kaikaivilu@gmail.com

\section{Citar como:}

Troncoso-Pérez, P., Nass, J., Gallegos, A., \& Alvarez, M. A. (2020). Entre la decolonización y el capital humano. reflexiones en torno al rol esperado del docente en el Chile post transición. Propósitos y Representaciones, 8(SPE1), e498. doi: http://dx.doi.org/10.20511/pyr2020.v8nSPE1.498 


\section{Resumen}

La relación entre movilidad socioeconómica y niveles escolares se ha tematizado desde la generalización de los sistemas institucionalizados modernos de educación. La exigencia de educación de calidad chilena está asociada al exitismo y a la formación de Capital Humano, estrellándose con desajustes entre políticas públicas, la crisis de legitimación de la educación pública, y el avance epistémico latinoamericano que influye el ejercicio pedagógico a través de la pedagogía crítica o la descolonización como nueva forma de organizar/re-crear conocimiento. Es así que el docente es requerido desde dos corrientes dicotómicas, lo que le convierte en un vórtice con inusitadas aristas. El presente artículo de reflexión busca exponer algunos aspectos de dichas contradicciones, abordando la teoría de Capital Humano en educación y de la decolonización de la pedagogía, en relación al rol esperado del profesorado en Chile.

Palabras clave: Rol docente esperado; Capital humano; Decolonización.

\section{Summary}

The relationship between socioeconomic mobility and school levels has been thematized since the generalization of modern institutionalized education systems. The demand for Chilean quality education is associated with exitism and the formation of Human Capital, crashing with mismatches between public policies, the crisis of legitimization of public education, and the Latin American epistemic advance that influences the pedagogical exercise through critical pedagogy or decolonization as a new way of organizing / re-creating knowledge. Thus, the teacher is required from two dichotomous currents, which makes him a vortex with unusual edges. This article of reflection seeks to expose some aspects of these contradictions, addressing the theory of Human Capital in education and the decolonization of pedagogy, in relation to the expected role of teachers in Chile.

Keywords: Expected Teaching Role; Human Capital; Decolonization.

\section{Introducción}

Chile es un país de discordancias diferenciado por sus políticas sociales y su modelo económico neoliberal de mercado que lo vuelven uno de los representantes de las deformaciones de la modernidad (Larraín, 2005), de la posmodernidad o en su defecto (Beck, 2002; Beck et al, 1997; MacLaren, 1997), de la macabra que puede ser la racionalidad instrumental como señala J. Habermas (1989).

Según datos del Banco Mundial, es un país con tasas de crecimiento constante que han desacelerado en los últimos años de un 6.1\% (2011) a $1.5 \%$ (2017); aun así fue una de las economías de más vertiginoso crecimiento en Latinoamérica, repercutiendo esto en la reducción de la pobreza (DSP, 2017). Se asocia este éxito - entre otras variables- a la adición de los años de escolaridad formal y la formación especializada para el trabajo (Brunner y Elacqua, 2003), que han acarreado el aumento sostenido de la demanda de acceso a la educación formal técnica/superior (Ottone \& Hopenhayn, 2000; Brunner et. al, 2005) y una carrera por los grados y espacios laborales.

Estas últimas características emanan de sociedades de riesgo (Beck et al, 1997), produciendo un fenómeno contrario a la esperada escolarización moral durkhemiana: el déficit de socialización (Tedesco, 1995), que implica la cosificación del docente, la pérdida de transcendencia de la familia en el traspaso de valores, un extendido sentimiento de anomia y la 
percepción constante de identidades sociales y nacionales amenazadas (Giddens, 1995; Bauman et al, 1996).

En este escenario, el rol del docente resulta impactado por las vicisitudes contextuales (Tedesco, 1995, Palermo, 2014). El desempeño profesional no solo está determinado por las características del profesor. Diversos autores tales como Bryk, Harding y Greenberg, 2012; Darling-Hammond, 2012; indican que la calidad de la enseñanza y los resultados del proceso educativo están fuertemente influenciada por el contexto de instrucción (Santelices et al, 2015) Es así que el rol esperado del docente reflecta, en este punto, las demandas aspiracionales y de transformación socioeconómica de la población.

Adicionemos que gracias a la crisis de la modernidad occidentalizante (Mignolo et al, 2014; Giddens, 1995; Bauman et al, 1996), en los últimos veinte años hemos presenciado la intensificación de la necesidad de generar alternativas epistémicas pertinentes para los territorios con historias de sometido o algún tipo de apremio ideológico. Latinoamérica despertará a la visualización de sus poblaciones originarias y su acervo, a la valoración de estas y la posibilidad de compendiar un cúmulo de saberes propios capaces de dar frente a la ola epistémica impuesta como única verdad (Robles, 2012; CLACSO, 2005). Nuevamente la pedagogía y la docencia no estarán exentas (Palermo, 2014); el rol del docente, semejante a la pedagogía crítica (McLaren, 1997), será exigido más allá de un animador cultural o un estimulador de la reflexión política (Freire, 2005), sino como un individuo además que debe ser capaz de repensar lo que sabe, rearticularlo en paisajes de opresión o descolonización de las estructuras conceptuales y ser-hacer partícipes a sus aprendiendo de dicha experiencia (Santorello, 2016).

Finalmente, las transformaciones habidas en los últimos cincuenta años en la arena política y en la economía global (Giddens, 1997; CLACSO, 2005) han repercutido en la planificación de los sistemas escolares (Bonal, 1998), generando que la escuela actúe como una especie de transmisor en el que podemos presenciar el devenir de dichas esferas de la vida cotidiana. Una de las crisis sufridas por estas esferas hizo comulgar a la escuela con la teoría de capital humano -y con una visión pragmática de la educación a través de la psicología educacional- arrojaron a los sistemas escolares (iniciando por el estadounidense), a la constante de educar para el trabajo y la movilidad socioeconómica, desplazando paulatinamente la aspiración inicial de la generación de ciudadanía (Puigross, 1999; Blanco, et Al, 2013).

\section{Elementos para situarnos en el contexto}

Las transformaciones culturales ocurridas durante las últimas décadas del s. XX llevaron a una nueva visión del sujeto (Tedesco, 1995; Giddens, 1995); producto de un creciente desarrollo económico en poblaciones antiguamente pobres (entre los años 2000 y 2015, la proporción de la población considerada pobre en Chile: US\$ 4 por día, disminuyó del 26 a $7.9 \%$; DSP, 2017) las que despertaron a las posibilidades del consumo (PNUD, 2017); sumado a la necesidad hegemónica propia de cualquier gobierno, convirtieron a la instrucción escolar en el aparato simbólico de inclusión, distribuidor de roles y posiciones sociales; fue y es un imprescindible bien simbólico (Ottone y Hopenhayn, 2000). A su vez, la merma en los índices de crecimiento en los últimos años (3\% de crecimiento del producto, similar a la llamada década perdida de los ' 80 ; Brunner, 2003) sumado a que los trabajadores sobreviviven a la precarización laboral, y un poco menos lo hace en situación de indigencia, con la peor distribución del ingreso entre las regiones del mundo (CLACSO, 2005), Chile y sus sistemas educativos se enfrentan a los desafíos de la revolución tecnológica y la globalización.

A pesar de los esfuerzos de las últimas décadas y el mayor gasto público la educación permanece rezagada a nivel regional (Brunner, 2011). La cobertura insuficiente y significativos niveles de exclusión y retención, por un lado, y escasa capacidad de compensar los déficits de capital cultural de los niños provenientes de los hogares más pobres, y bajos logros en el aprendizaje, por otro, se truecan en un gasto ineficiente (UNESCO, 1998). 
Aun así, las percepciones de la población respecto del estado del sistema escolar, como comenta Bellei (2015), proyectan un complejo cuadro con elementos convergentes y divergentes de diagnóstico y expectativas (Manzi \& Garcia, 2016; Redón, 2011).

\section{Algunos ejes Conceptuales}

Son tres las nociones centrales a abordar, que en cierta forma ya hemos introducido con anterioridad. Estos son el concepto de rol esperado del docente, algunos elementos del capital humano en educación y los aportes de la pedagogía decolonial. Evidentemente ante dos posturas opuestas en gran medida, el articulador de ambos campos de ideas es el rol docente.

Tenemos así que el rol consiste en la función ejercida en los sistemas sociales, asignado por las particularidades históricas y territoriales, e impuesto de manera coercitiva o tácita en los sujetos (Giddens, 2010). Cumple con asegurar la cohesión social (Durkheim, 2002) y la reproducción económica propia de las sociedades modernas (Tedesco, 1995; Bourdieu et al, 2008). Existen roles ideales, que son aquellos a los que aspira la población, o que se espera idealmente su cumplimiento; y roles desempeñados, que es la ejecución efectiva del rol, con todos los elementos externos que inevitablemente le afectan (Giddens, 2010).

Como se señala, las características de los roles son asignadas por la población a través de diversos dispositivos legitimizados culturalmente: Familia, Escuela, Medios de comunicación, etc. (Puigross; 1999; Bonal, 1998) en todos los casos, el rol depende las aspiraciones proyectadas sobre la función, del que emerge una "imagen" de lo esperado (Bourdieu et al, 2008, Bourdieu, 2002). Dicho reflejo o idea resulta un medio eficaz de construcción de conciencia colectiva (Durkheim, 2002; Tedesco, 1995). Saussure, Pierce, Barthes, Eco y Greimas, entre otros, lo ejemplifican en sus estudios de lingüística, filosofía y semiología, el camino entre el objeto y la mente (Zecchetto, 2013). En otro extremo, para A. Gramsci (1976), es una forma de hegemonización impuesta no solo por la dominación eterna o material, sino también cultural, que genera la subordinación.

En cuanto al rol del docente, históricamente ha sido empapado del entorno e inevitablemente utilizado como dispositivo de poder que articula las masas (Foucault, 1991; Puigross, 1999) y por su lucha por la autonomía (Freire, 2005; Palermo, 2014), no exenta de contradicciones (Reyes, et Al., 2012). Extensa ha sido la trasformación de sus funciones (Bedoya, 2005; Blanco et al, 2013), pero la que nos atañe es la producida en la llamada tercera revolución educativa (Esteve, 2003): Escolarización inclusiva, déficit culturales, y el 100\% de cobertura escolar, que se traducen en la visibilización y abordaje por parte del docente de problemáticas sociales, multiculturales, morales, entre otros, en un espacio escolar en que se deben integrar (en el aula) episodios de delincuencia, abusos, carencias emocionales, en un momento socio-histórico en el que la humanidad se desdibuja cosificado por el mercado (Bauman, 2007).

Por otra parte, el sistema educacional chileno figura con tres ejemplares de escuelas: municipales (públicas), particulares subencionadas, y privadas (MINEDUC, 2017). Para el año 2008 había aproximadamente 11.907 escuelas en Chile, 49\% de las cuales eran municipales, 44\% subvencionadas y $6 \%$ privadas; con 176.500 profesores en aula, de los cuales $55 \%$ se desempeñaban en escuelas municipales (Santelices, et. Al. 2015; MINEDUC, 2017). Sus salarios están relacionados al sueldo mínimo indicado por el Estado, antigüedad, bonos por capacitación, asiento geográfico y responsabilidades administrativas, y de bonos que dependen de la acreditación de excelencia (Sistema Nacional de Evaluación del Desempeño de los Establecimientos Educacionales Subvencionados, SNED), y una certificación de excelencia de desempeño (Asignación de Excelencia Pedagógica, AEP) (MINEDUC, 2017).

El Marco para la buena Enseñanza establece lo que todos los docentes chilenos deben conocer, saber hacer y ponderar para determinar cuán bien desempeñan su trabajo, sin embargo, y como se expuso con anterioridad, más allá de las implicancias formales del desempeño docente, 
se encuentra la impronta cultural, determinada por la coyuntura, sobre cuál y cómo debe desplegarse el rol docente.

En términos generales, la docencia se establece como un trabajo que envuelve un compromiso social y político en la búsqueda de una mayor justicia social (Sisto, 2012: Torrealba, 2006). Sin embargo, la baja valoración social de la profesión, salarios bajos, exceso de trabajo administrativo, un currículum sobredimensionado, una formación inicial insuficiente y carreras de pedagogía poco selectivas, señalan que se espera poco de los docentes (Brunner, 2005a), y de que terriblemente los resultados de los alumnos están más determinados por su nivel socioeconómico que por la calidad de su educación (Tedesco, 1995).

Abordando el segundo eje conceptual tenemos a la teoría de Capital Humano. Para abordar sus contenidos debemos explicar las instancias en que llega a los sistemas escolares. Con ese propósito debemos recordar la crisis de escolarización sufrida en Estados Unidos a mediados del s. XX que significó el quiebre del Pacto Ciudadanía -Estado (escolarización por movilidad socioeconómica), la caída de los modelos escolares funcionales tecnocráticos (con el consecuente desprendimiento de las responsabilidades de los Estados modernos, al menos en los niveles superiores de educación, la distribución del trabajo y es estatus) y la necesidad de un nuevo trasfondo conceptual para amparar el sistema escolar y que además fuera acorde a la economía de mercado que se desplegaba exitosa en aquellos años (Bonal, 1998).

La teoría de Capital Humano proviene de las esferas de la economía y la administración de empresas. Su idea central es que tematiza al ser humano como fuente inagotable de recursos y que el capital humano fue el factor oculto que no se había tomado en cuenta en la función de la producción hasta la fecha. Políticamente es demostrativa (legitima la razón técnica sobre la tradicional); ideológicamente opone crecimiento a desarrollo (a favor del primero), por ende, lo cuantitativo sobre la cualitativo. Psicológicamente aporta valores racionales y la inculcación de la motivación por el rendimiento. No es de extrañar que para esta matriz teórica la instrucción formal sea considerada un bien capital (Bonal, 1998; Brunner, 2003).

Entre sus representantes se encuentra Theodore W. Schultz (1983), economista estadounidense que planteó el uso eficiente de los recursos humanos y la igualdad de oportunidades para beneficio del mercado. Otro representante es Gary Stanley Becker, también economista estadounidense; propuso que la educación y la formación superior son formas de inversión que realizan individuos racionales que buscan incrementar su eficiencia productiva y sus ingresos. El agente económico (individuo) al decidir entre invertir o no (seguir estudiando o no) arbitra entre los beneficios y costos de inversión. Seguirá estudiando si el valor actualizado neto de los costos y de las ventajas es positivo; invertirá para sí mismo y esa inversión se realizará en base a un cálculo de riesgo. El Hombre se convierte en su propia empresa; y sobre la formación general, adquirida en el sistema educativo dirá que incrementa la productividad media y marginal en la economía. El costo se traspasa a los individuos o a algún organismo público (Brunner; 2003; Bonal, 1998).

La Teoría de capital Humano propone que la educación puede ser considerada como cualquier otra forma de capital físico, y que es una inversión que proporciona una tasa de rentabilidad determinada; que existe una relación positiva entre desarrollo económico y tasas de escolaridad en todos los niveles productivos, y por ende una relación positiva entre años de estudio e ingresos, lo que sería reflejo de los efectos de la educación sobre la productividad (utilizan el Modelo de Mincer ${ }^{1}, 1974$ para justificar); que los individuos pueden conocer el rendimiento a obtener según su inversión educativa y que la desigualdad social se explicaría como resultado lógico del aprovechamiento individual de las oportunidades o como expresión de distintos sistemas de preferencias; y finalmente que a mayor educación, mayor es el avance

\footnotetext{
${ }^{1}$ Ecuación Mincer: Modelo semi-logarítmico articulado como variable dependiente el logaritmo de los
} ingresos y como variables independientes los años de educación y la experiencia laboral (Bonal, 1998) 
tecnológico/productivo, y mayor es el crecimiento económico; su implementación auguraba mejores relaciones interpersonales, bajas en las tasas de criminalidad, y efectos redistributivos al tratarse de una alta inversión de gasto público.

En el caso de Chile, un representante de esta corriente es Brunner (2005) que visualiza la escuela como ente para superar la pobreza y reutliza el modelo de logro de los sistemas funcionales para estimular la competencia entre estudiantes por los puestos de trabajo por medio de la instrucción formal (Brunner, 1993, Brunner \& Elacqua, 2003; Brunner, 2011) El sistema escolar chileno formaría así capital humano calificado. Entre sus ideas se encuentran que la evidencia la productividad es el cálculo de los rendimientos privados de las inversiones en educación, lo que explicaría las diferencias de ingresos según distintos niveles de escolaridad; que la revolución tecnológica requiere de ejércitos de especialistas; y que el sistema educativo entrena y selecciona, por lo que debe expandirse (Brunner \& Elaqua, 2003; Brunner et al, 2005).

A medida que las sociedades transfiguran su base económica y buscan modernizarse tecnológicamente, necesitan también mejorar el perfil de su fuerza de trabajo (Brunner \& Elaqua, 2003). Bajo esta concepción, todos seríamos capitalistas: unos poseen el capital económico y otros el humano, por lo que las desigualdades son una responsabilidad individual.

Concluyendo los ejes, encontramos la teoría decolonial, pero en el ámbito pedagógico. Primero abordaremos algunas nociones centrales de esta matriz teórica.

Entre los exponentes se encuentran Boaventura de Sousa Santos, quien elabora una revisión crítica del colonialismo del saber en América latina (2009), y su incidencia en las instituciones modernas (Mignolo et al, 2014); a Catherine Walsh que revalora las otras Ciencias, otros conocimientos, saberes idénticamente importantes y aportativos que el acervo occidental, y en particular, que la fuente de este saber no se encuentra en el curriculum formal, sino que en nuestros territorios, en nuestras esferas de vida y tradiciones de pueblos originarios, propone así la comunión entre los saberes, una complementariedad respetuosa y equitativa (Walsh, 2007); y Rivera Cusicanqui (2016), que propone la Mirada Periférica y la Percepción corporal para conocer/ ser el territorio propio y otros de manera comprometida, donde el saber guarde sentido práctico con la realidad.

Para Mignolo (2003; 2011) la colonialidad es hermana de la modernidad por sus supuestos redencionistas; en el extremo opuesto se encuentra lo que no buscó redención, y por tanto, fue sometido y silenciado. Este acallar con el tiempo acumuló desazón y finalmente desató resistencia: la descolonización.

La implementación en contextos prácticos de estos pensamientos podemos revisarlo a través de la pedagogía decolonial, que pretende desmantelar los mandatos de la modernidad y su lado oculto que es la colonialidad (Palermo, 2014). Alientan nuevas formas de acción política e insurgencia, a la vez que construyen alianzas, esperanzas y otras visiones de estar en sociedad, relacionadas con sus pertinencias territoriales (Sartorello, 2016). A la vez buscan otra relación con la naturaleza, y apoyan el proyecto social, ético y político de la interculturalidad crítica.

Entre sus pensadores basales se encuentra Frantz Fanon (2010) y sus Condenados de la Tierra, donde acusa que la deshumanización es un componente central de la colonización, por lo que la humanización requiere de la descolonización, y su vehículo es la pedagogía. A diferencia de Freire visibiliza el lugar de la opresión como un lugar racializado; para él la colonización deja de denostar al colonizado, cuando este último se revela o admite la supremacía de los valores occidentales, por ende, el espacio escolar pasa a ser un escenario en donde se enfrentan ambos mundos y del que deben evidenciarse. Los elementos esenciales que aporta son aproximaciones a la totalidad (realidad social comunitaria), la estructura (como eje del devenir y como generador de opuestos) y la historicidad, espacio donde se plasman las anteriores y los conflictos emanados de la segunda. 
La decolonización comprende así una forma de (des) aprendizaje o desprendimiento (Palermo, 2014): desaprender todo lo impuesto y asumido por la colonización y la deshumanización para aprender a ser hombres y mujeres. El maestro tiene la responsabilidad de ayudar activamente en ese despertar, y, por ende, la educación política significa abrir las mentes, despertar y permitir el nacimiento de la inteligencia con relación al mundo. Es educar a los seres humanos para ser accionales, para actuar por sí mismos, es facilitar la formación de subjetividad y autoreflexión para una praxis de liberación.

\section{Aportes para la discusión}

Desde la década de los ochenta el gobierno chileno, la intelectualidad más conservadora (y no tanto), los medios de comunicación, y no pocos organismos internacionales como la OCDE, el Banco Mundial o la Unesco, promovieron a la educación como el principal agente para el desarrollo de los países (Bellei, 2015).

Desde el punto de vista de la percepción de la gente la educación aparece como uno de los bienes sociales más preciados, si no como el medio más potente para alcanzar el bienestar individual y colectivo (Brunner, 2003; Redón, 2011). No resulta extraño verificar que la población manifiesta una creencia creciente respecto al papel que la educación en las mejoras materiales y la movilidad social (DSP, 2017), ilusión que explica en el mejoramiento masivo de las condiciones de vida en el país (Blanco, et Al. 2013; PNUD, 2017).

Con la dictadura militar de telón, se implementó en 1980 la Ley General de Educación (LEGE), desde entonces el país implementó decididamente un modelo escolar acorde con el económico, y ese fue paulatinamente el de la T.C.H.; un modelo de mercado para la entrega de servicios educacionales, descentralizado (municipalización), incorporando prestadores privados de servicios (Corvalán, 2013). El plan era generar un mercado de escuelas competitivas sostenido por el subsidio a la demanda, según el discurso, espíritu y estímulo del mejoramiento de la calidad, profesionalización docente y fin de la docencia normalista en aula.

Desde 1990, se inicia una reforma con fines de mejora principalmente estructurales y didácticos (proyectos MECE—, Enlaces, etc.), como así también de transformación curricular (formación en función de las competencias, habilidades y conocimientos). En este contexto, desde el año 2000 se ha puesto atención en generar estándares de ejercicio para docentes, asumiendo que cumpliendo aquellos, aumentará la calidad educativa medida a través del Sistema de Medición de Calidad de la Educación (SIMCE) (MINEDUC, 2017). A través de la política de evaluación e incentivos al desempeño, se espera modelar el desempeño del profesorado.

Por consiguiente, se establece una relación directa entre éxito en pruebas estandarizadas y aumento del salario profesional, lo que por el lado amable puede ser visto como un premio al esfuerzo profesional, pero en el aspecto humano como la cosificación del desempeño pedagógico. Si bien dicha dehumanización se produce desde fuera del aula (Blanco, et. Al., 2013), claramente es potenciada a través de espacios como el sistema educativo (Bourdieu, 2002).

El rol exigido al docente desde la cultura nacional, por tanto, queda suscrito a un reproductor en un espacio que fabrica puntajes, más que una persona que forma jóvenes. Ese aspecto se plasma en las necesidades que la población manifiesta en las movilizaciones nacionales, en las escuelas y en las mismas universidades. La pugna de qué se espera del profesor, y que busca formar está en la encrucijada de una sociedad de libre mercado, en su mayoría muy cómoda en su escaso poder de consumo (PNUD, 2017), que le demanda estudiantes exitosos y proactivos, más allá de personas que puedan aportar comunidad. Rol impuesto que no todos los docentes aceptan tan abiertamente (Reyes et al., 2012).

Entre las variables de impacto sobre el aprendizaje los docentes guardan especial protagonismo (Reyes et al, 2012; Manzi \& Garcia, 2016). Determinantes son la formación inicial 
y el desempeño en el aula, el tipo de comunicación que instauran con los alumnos, el clima de aprendizaje genera, etc. Se enfrentan además a sobrecarga de trabajo y excesivas horas de planificación, incluida la precariedad de recursos (materiales, humanos, financieros) que evidencia la incongruencia entre lo ideal (desde la política estatal, ministerial, municipal) y las condiciones reales para el ejercicio de la práctica docente (Reyes et al, 2012; UNESCO, 2013).

No es de extrañar nuevamente, que como señala Tedesco (1995), se produzca el déficit de socialización, esa falta de hegemonía, no subordinada, sino comunitaria, producida por la ineficacia de las instituciones normativas centrales: familia y escuela, producida por la vorágine de la vida capitalista, con el deterioro evidente de la valorización del docente.

Por otra parte, la relación positiva entre educación y productividad del trabajo está latamente negada a través de estudios realizados desde la década del setenta, pero se insiste en su efectividad en países como Chile. Los salarios no han sido determinados en base a la productividad, y por ende, tampoco dependen totalmente de la cualificación del trabajador. A su vez, la distribución de las posiciones educativas, de los estatus ocupacionales y de los ingresos tampoco se relacionan directamente, actuando vectores como la tradición, los contactos o hasta la misma suerte en el éxito escolar y laboral. Por ende, no afecta la movilidad social: origen social familiar tienen más incidencia (Bourdieu, 2002; PNUD, 2017).

En esta encrucijada, donde el profesorado se encuentra estimulado por el mismo sistema escolar al que pertenece, exigido por las familias de sus pupilos, y refrendado por una amplia base conceptual académica -científica que lo avala- emerge esta concepción completamente opuesta, la pedagogía decolonial, avalada por otro espectro de la academia, pero a la vez por las mismas identidades negadas por años de imposición epistémica, por las historias de ellos mismos ¿Qué podrá resultar de todo aquello?

Sin embargo, y a pesar de las oportunidades que ofrece la pedagogía decolonial para rearticular desde otro punto la escuela latinoamericana, la independencia política, debe apoyarse en unas bases económicas sólidas, y aun así la ruptura de los vínculos de dependencia, por muy importante que sea, sólo es una de las etapas de la descolonización.

Por otra parte, y reconociendo las formas que tiene la economía capitalista para transformarse y readaptarse, es inclusive posible que la misma noción de decolonialidad sea absorbida de manera parcial e instrumentalizada por esos mismos campos de docencia pragmática. Ya lo señalaba L. Althusser, los docentes son héroes buscando mejorar sus espacios disciplinares, más lo único que hacen en el marco de este modelo económico, es hacer más eficientes los aparatos ideológicos de opresión.

\section{Reflexiones finales}

La sociedad chilena, contractualizada, atomizada, sin mucho fondo en común, comparado con las etnias presentes en el territorio, encuentra dificultad para cohesionarse; se concibe así misma en un mundo multidimensional, momentáneo y autoreferente. Esto plantea un desafío adicional para la escuela, en un contexto social cuyas bases tradicionales se han debilitado. Las interrogantes que esto abre son apremiantes, como el mismo rol del docente. La educación en su función económica, recepta las teorías desarrollistas de la educación (formación, selección y clasificación de la mano de obra) por un lado, y aplaude las novedades epistémicas incorporadas al aula a través de la validación de lo otro como conocimiento pertinente. Los peligros de caer en la folclorización son inminentes.

Si bien la educación no asegura movilidad social ascendente ni la reducción de las desigualdades sociales, no hay oportunidad sin educación. Es necesario redirigir la escolarización hacia un campo más particularista y crítico, que asegure el aprendizaje reflexivo, pertinente al territorio más que al mercado. 
Por otra parte, algunos docentes han adoptado formas de resistencia propias contra la institucionalidad escolar, posicionándose como actores y víctimas de ésta y del silenciamiento político desde la imposición institucional de la profesionalización docente. Construir sobre bases nuevas/viejas, hasta entonces orientadas en función de las necesidades o de los beneficios del colonizador. El afianzamiento de una identidad personal, como cimiento en la forma de relacionarse (alteridad) para construir lo común y el sentido valorativo de bien común como lo político, requieren de un docente capaz de inspirar en este gran desafío de transformación socialpersonal.

Finalmente, el sistema escolar reflecta las transformaciones que experimenta el contexto cultural en que está inmersa; sin saber qué predominará, resulta más humano esperar que prime la contextualización de los significados que le permiten ser lo que conocemos, alumnos, docentes, familia y comunidad: Escuela y el "buen vivir".

\section{Referencias}

Banco Mundial (2017) Chile - Diagnóstico Sistemático de País: La Evolución hacia una Sociedad más próspera (DSP). Systematic Country Diagnostic. $\mathrm{N}^{\circ} 107903$.

Bauman, Z., Luhmann, N., \& Beck, U (1996). Las consecuencias perversas de la modernidad. J. Beriain (comp.). Barcelona: Anthropos.

Bauman, Z. (2007). Los retos de la educación en la modernidad líquida. Barcelona: Gedisa.

Beck, U. (2002). La sociedad del riesgo: hacia una nueva modernidad. Barcelona: Paidós.

Beck, U. Giddens, A., \& Lash, S. (1997). Modernización Reflexiva. Política, tradición y estética en el orden social moderno. Madrid: Alianza.

Bedoya, J. (2005). Epistemología y pedagogía: Un ensayo histórico crítico sobre el objeto y método pedagógicos. Bogotá: Ecoe.

Bellei, C (2015). El gran experimento. Mercado y privatización de la educación chilena. Santiago. Lom.

Blanco, E., Cárcamo, H., \& Ponce de León, R. (2013) Sociedad, Cultura y Educación. Chillán. Ediciones UBB.

Bonal, X. (1998). Sociología de la educación: Una aproximación crítica a las corrientes contemporáneas. Barcelona: Ediciones Paidós Ibérica.

Bourdieu, P. (2002). Capital cultural, escuela y espacio público. México: Ed. Siglo XXI.

Bourdieu, P., \& Passeron, Ch. (2008). La Reproducción. Elementos para una teoría del Sistema de Enseñanza. Madrid: Editorial Popular.

Bryk, A., Harding, H., \& Greenberg, S. (2012). Contextual influences on inquiries into effective teaching and their implications for improving student learning. Harvard Educational Review, 82(1), 83-106.

Brunner, J.J., \& G. Elacqua (2003). Capital humano en Chile. Santiago, Chile: La Araucana.

Brunner, J.J. (2011). La lucha por la formación de las elites: campo y canales formativos. Revista UDP, 9.119-143.

Brunner, J.J., Elacqua, G., \& Tillett, A. (2005). Guiar El Mercado. Informe Sobre La Educación Superior en Chile. Santiago: Universidad Adolfo Ibáñez.

Brunner, J. J. (2005a) Educación en Chile: el peso de las desigualdades. Conferencias Presidenciales de Humanidades. Santiago de Chile, 20 de abril 2005.

Brunner, J.J. (1993). El Caso Chileno, Evaluación Académica, CRE - UNESCO, Vol. 2, París, (pp. 37-61).

Consejo Latinoamericano de Ciencias Sociales (2005). Las reformas educativas en los países del cono sur: Un balance crítico. Buenos Aires: CLACSO.

Corvalán, J. (2013). La narrativa educacional chilena y su proceso de transformación reciente: un análisis sociológico-histórico. Folios, 37, 63-81.

Darling-Hammond, L. (2012). Powerful teacher education: Lessons from exemplary programs. John Wiley \& Sons.

De Sousa Santos B. (2009). Una epistemología del sur. México: CLACSO - Siglo XXI.

Durkheim, E. (2002). La educación moral. Madrid: Trotta. 
Esteve Zarazaga, J.M. (2003). La tercera revolución educativa: La educación en la sociedad del conocimiento. José Manuel. Barcelona: Paidós Ibérica.

Fanon, Frantz (2010). Los condenados de la tierra. México: F.C.E.

Foucault, M., Donzelot, J., Grignon, C., de Gaoudemar, J., Muel, F., \& Castel, R. (1991). Espacios de poder. Madrid: Ediciones Endymion.

Freire. P. (2005). La importancia de leer y el proceso de liberación. México D.F.: Siglo XXI.

Giddens, A (2010) Sociología. Madrid: Alianza.

Giddens, A. (1995). Modernidad e identidad del yo. Barcelona: Península.

Giddens, A., Beck, U., \& Lash, S. (1997). Modernización reflexiva: política, tradición y estética en el orden social moderno. Alianza Editorial.

Gramsci, A. (1976). Maquiavel, a política eo Estado moderno. Rio de Janeiro: Civilização Brasileira.

Habermas, J., \& Redondo, M. J. (1989). Teoría de la acción comunicativa: complementos y estudios previos. Madrid: Cátedra.

Larraín, J. (2005). ¿América Latina moderna? Globalización e identidad, Santiago: LOM.

Manzi J., \& García, M. (2016), Abriendo las puertas del aula. Transformación de las prácticas docentes. Santiago de Chile: Ediciones U.C.

McLaren, P. (1997) Pedagogía crítica y cultura depredadora: políticas de oposición en la era posmoderna. Barcelona: Paidós.

Mignolo, W. (2011). La colonialidad a lo largo y a lo ancho: el hemisferio occidental en el horizonte colonial de la modernidad. En Edgardo Lander (compilador), La colonialidad del saber: eurocentrismo y ciencias sociales. Perspectivas latinoamericanas (pp. 73104). Buenos Aires: Clacso.

Mignolo, W. (2003). Un paradigma otro/colonialidad global, pensamiento fronterizo y cosmopolitismo crítico. En Historias locales/diseños globales. Colonialidad, conocimientos subalternos y pensamiento fronterizo, (pp. 19-60). Madrid: Akal.

Mignolo, W., García, A., \& Walsh, C. (2014). Interculturalidad, descolonización del Estado y del conocimiento. Buenos Aires: Ediciones del Signo.

Ministerio de Educación. (2017). Informe Nacional. Revisión de Políticas Educativas en Chile. Stgo de Chile: Centro de estudios MIDEDUC.

Ottone E., \& Hopenhayn, M. (2000). El gran eslabón: educación y desarrollo en el umbral del siglo XXI. México: Fondo de Cultura Económica.

Palermo, Z. (Ed). (2014). Para una pedagogía decolonial. Buenos Aires: Ediciones del signo.

PNUD. (2017). Desiguales. Orígenes, cambios y desafíos de la brecha social en Chile. Santiago de Chile: Uqbar editores.

Puigross, A. (1999). Educar, entre el acuerdo y la libertad. Buenos Aires: Planeta.

Redón Pantoja, S. (2011). Escuela e identidad: Un desafío docente para la cohesión social. Polis (Santiago), 10(30), 447-476.

Reyes L., Cornejo R., Arévalo A., \& Sánchez R. (2012). Ser docente y subjetividad histórica en el Chile actual: discursos, prácticas y resistencia, Polis (En línea), 27. Recuperado de http://journals.openedition.org/polis/901

Rivera Cusicanqui, S., Domingues, J., Escobar, A., \& Leff, E. (2016). Debate sobre el colonialismo intelectual y los dilemas de la teoría social latinoamericana. Cuestiones de sociología: Revista de estudios sociales, 14, 1668-1584.

Robles F. (2012) Epistemologías de la modernidad: entre el etnocentrismo, el racionalismo universalista y las alternativas latinoamericanas. Cinta de Moebio, 45.

Santelices, M. V., Galleguillos, P., González, J. \& Taut, S. (2015). Un estudio sobre la calidad docente en Chile: El rol del contexto en donde enseña el profesor y medidas de valor agregado. PSYKHE, 24(1), 1-14. https://doi.org/10.7764/psykhe.23.2.673

Santorello, S.C. (2016). Política, epistemología y pedagogía: El método inductivo intercultural en una escuela tseltal de Chiapas, México. LiminaR, 14(1), 121 - 143.

Sisto, V. (2012). Challenged Identities: Individualization, Managerialism, and Teaching Work in Chile Today. Psykhe (Santiago), 21(2), 35-46. Recuperado de https://scielo.conicyt.cl/scielo.php?script=sci_arttext\&pid=S0718$\underline{22282012000200004 \& \operatorname{lng}=\mathrm{es} \& \operatorname{tn} \mathrm{ln}=\mathrm{en}}$. 
Schultz, T. W. (1983). Inversión en capital humano. Educación y sociedad, 8(3), 180-195.

Tedesco, J. C. (1995). El nuevo pacto educativo. Madrid: Ed. Anaya.

Torrealba, T. (2006). El sentido común de los maestros: algunos problemas epistemológicos. Revista mexicana de Investigación Educativa, 16(7).

UNESCO (1998). Informe Mundial sobre la Eeducación: Los docentes y la enseñanza en un mundo en mutación; Madrid: Santillana/Ediciones UNESCO.

UNESCO (2013) Antecedentes y criterios para la elaboración de políticas docentes en América Latina y el Caribe. Stgo - Chile: OREALC.

Walsh, C. (2007) ¿Son posibles unas ciencias sociales/culturales otras? Reflexiones en torno a las epistemologías decoloniales. Revista Nómadas, (26), 102-113.

Zecchetto, V. (2013). Seis semiólogos en busca del lector. Abya-Yala/UPS. 